\title{
Preface to the special issue on finite geometries
}

\author{
Ilaria Cardinali ${ }^{1} \cdot$ Michel Lavrauw ${ }^{2} \cdot$ Klaus Metsch $^{3} \cdot$ Alexander Pott $^{4}$
}

Published online: 8 January 2019

(c) Springer Science+Business Media, LLC, part of Springer Nature 2019

The present volume contains a collection of research papers in the thriving area of Finite Geometries. This includes the topics: Combinatorial structures in Galois geometries; Finite incidence geometry; Algebraic curves and varieties over finite fields; Geometric and algebraic coding theory; Finite groups and geometries; Algebraic design theory.

Geometry is one of the central fields within mathematics. Historically, geometry firstly dealt with objects from real space. Finite geometry started, when the concepts of points and lines were translated from the continuous to the discrete world. One way to make this explicit is by starting with a geometry over the reals and replacing the field of real numbers by a finite field, thus obtaining a discrete object. The resulting geometry obtained from real affine and projective spaces is what is commonly referred to as Galois geometry. The axiomatic approach to geometry also contributed to this new point of view, since it allows geometries consisting only of a finite number of objects, which are not immediately obtained from affine or projective spaces over fields. One thus quickly arrives at the subject of combinatorial designs. Besides the fact that designs are beautiful objects in their own right, they also have many applications, for instance in coding theory, data storage or cryptography. To some extent, finite geometry can be viewed as the part of design theory which deals with objects constructed via finite algebraic structures such as finite groups or finite fields.

This volume collects several papers which discuss various emerging aspects of finite geometry.

One paper deals with a rather recent topic in finite geometry, namely the $q$ analogues of classical combinatorial problems like the Erdős-Ko-Rado theorem (Karen Meagher).

$凶$ Michel Lavrauw

mlavrauw@sabanciuniv.edu

Ilaria Cardinali

ilaria.cardinali@unisi.it

Klaus Metsch

kmet@math.jlug.de

Alexander Pott

alexander.pott@ovgu.de

1 University of Siena, Via Roma, 56, 53100 Siena, Italy

2 Sabancı University, Orta Mahalle, 34956 Tuzla, Istanbul, Turkey

3 Mathematisches Institut, Justus-Liebig-Universität Gießen, Arndtstraße 2, 35392 Gießen, Germany

4 Otto von Guericke University, 39106 Magdeburg, Germany 
Difference sets and their generalizations are a central topic in finite geometry, and they are the subject of two papers (Marco Buratti; Stefaan de Winter and Zeying Wang). Difference methods are also used in the paper by Marco Buratti and Francesca Merola, which deals with a fairly new problem on finite geometry, of a more combinatorial flavor.

Hjelmslev planes (that have been used recently to construct very good codes) are the topic of the paper by Ivan Landjev.

Arcs and codes (two closely linked objects, for instance through the MDS conjecture), are the theme of the two papers by Daniele Bartoli, Massimo Giulietti and Maria Montanucci, and by Cunsheng Ding, Stefaan de Winter and Vladimir Tonchev.

Classical questions about codes derived from geometries are discussed by Fernando Piñero and Prasant Singh (weight distribution), by Dieter Jungnickel, Spyros Magliveras, Vladimir Tonchev and Alfred Wassermann, and by Assia Rousseva (minimum distance). Motivated by applications in flash memories, an interesting variation of classical codes are discussed in a paper by Igor Shparlinski and Arne Winterhof.

Blocking sets have been the object of interest by many researchers throughout the last decades, and they are also contained in this volume (Aart Blokhuis, Leo Storme and Tamasz Szőnyi). A closely related subject is the study of the set of directions determined by a set of points in the affine space (Jan De Beule, Jeroen Demeyer, Sam Mattheus and Péter Sziklai).

Generalizing properties of sets of points to properties of sets of subspaces (e.g. being in general position) leads to various interesting objects in higher dimensional projective spaces. An example is the paper by Hiroaki Taniguchi in which $d$-dimensional dual hyperovals constructed from semifields are studied.

Embeddings of point-line geometries are the subject of the paper by Bart De Bruyn, studying homogeneous embeddings of dual symplectic polar spaces.

Last but not least, there is a paper dealing with classical algebraic varieties in projective spaces over finite fields. The paper by Anton Betten and Fatma Karaoğlu studies cubic surfaces with 27 lines over finite fields.

The special issue was preceded by the international conference Finite Geometries, 5th Irsee Conference (September 2017 in Irsee, Germany). The articles have undergone rigorous peer-review according to the journal's high standards. There have been 18 submissions to this volume, of which 16 have been accepted. These papers reflect the diversity of the type of problems which are currently being investigated in the area of Finite Geometries.

Publisher's Note Springer Nature remains neutral with regard to jurisdictional claims in published maps and institutional affiliations. 